\title{
Crashworthiness response of honeycomb metallic-GFRP energy absorption devices
}

\author{
L. Romera, J. Paz, M. Costas, J. Díaz \& S. Hernández \\ Structural Mechanics Group, School of Civil Engineering, \\ Universidade da Coruña, Spain
}

\begin{abstract}
In this research, an original design of an impact absorber made of a metallic tube with an inner honeycomb-shaped part made of a glass-fiber reinforced polyamide is analyzed. For this task, we have employed the explicit module of the finite element code ABAQUS. The aim is to assess the crashworthiness response of this complete specimen, varying different parameters. For the honeycomb, cell size and wall thickness are the two factors that are changed; whereas only the wall thickness in the metallic tube is variable. The honeycomb height can also be modified, being always equal or smaller than the height of the metallic tube. Two functions are selected as metrics to evaluate the performance of the designs. These functions are the specific energy absorption of the specimen (SEA) and the peak load $\left(P_{\text {peak }}\right)$ produced during the crushing of the part. We have obtained quality results which allow a deep understanding of the crash performance of the absorber.

Keywords: Crashworthiness, impact absorbers, honeycomb structure, glass fiber, finite elements.
\end{abstract}

\section{Introduction}

Honeycomb structures are well known for their excellent energy absorption characteristics and their light weight. The first studies are dated in 1963, when the NASA granted an investigation by R. K. McFarland on the post-buckling behavior of hexagonal cell structures [1]. Since then, this design has been deeply looked into, both theoretically and experimentally [2-7], proving to be one of the best shapes when $\mathrm{s}$ lightweight design of crash absorbing structures is pursued. Regarding the materials selected for the construction of the honeycomb structure, a general use of aluminum is observed. Its lightness and ductility make it very 
suitable for a structure aimed to absorb as much energy as possible with a reasonable weight. These aluminum honeycomb structures can even be filled with artificial foams, combining the their stable collapse with the ductility of aluminum $[8,9]$. However, to the author's knowledge, little is known regarding the usage of materials such as glass or carbon fiber reinforced polymers to build these hexagonal absorbing structures. These kind of materials shows a fragile fracture behavior which a priori is undesirable. However, if they are combined with the ductile and progressive collapse of a metal part, the combination can offer higher values of absorbed energy for a very minor increase of the overall weight. The mean crushing strength of a metal tube filled with a honeycomb structure is therefore increased when compared to the empty one [10]. Therefore, complementing the metal tube with a core material translates into a better crashworthiness of the specimen, since the energy absorption values are higher and the overall weight of the car can be reduced.

Since the reduction of the part's mass is necessary, the absorber must be carefully designed. It is essential to understand how geometrical variations in the honeycomb part play an important role on the final performance of the element. This research offers an insight on the sensitivity of the absorber's crashworthiness to variations of its geometries.

\section{Design of the absorber and construction of the finite element model}

The proposed design consists of a 25-centimeter long square hollow section steel tube filled with a honeycomb structure made of a glass-fiber reinforced polyamide. This element is crushed at a constant speed of $64 \mathrm{~km} / \mathrm{h}(17.78 \mathrm{~m} / \mathrm{s})$, matching the Euro NCAP standard for frontal impact tests [11] up to a crushing length of $\delta$ equal to 18 centimeters.

Four design variables (table 1) have been selected, varying the geometry of the absorber. These are:

T1 Thickness of the steel sheets.

T2 Thickness of the GFRP honeycomb reinforcement.

D Diagonal length of a single honeycomb cell.

H1 Height of the GFRP honeycomb reinforcement.

Some views of the specimen are offered in figures 1 and 2 , in which the variables are labeled. Table 1 gives insight on the upper and lower bounds of each variable, as well as their initial values and to which specimen part they belong to. Regarding the materials, the selected steel for the outer tube is a standard S-275 J0H steel, which is modeled through a Johnson-Cook strain-rate sensitive model [12]. Taking into consideration the strain rate is essential, since different parts of the tube are loaded at very different load rates during the analysis. The constitutive equation of 
Table 1: Characteristics of design variables from figures 1 and 2. All dimensions in millimeters.

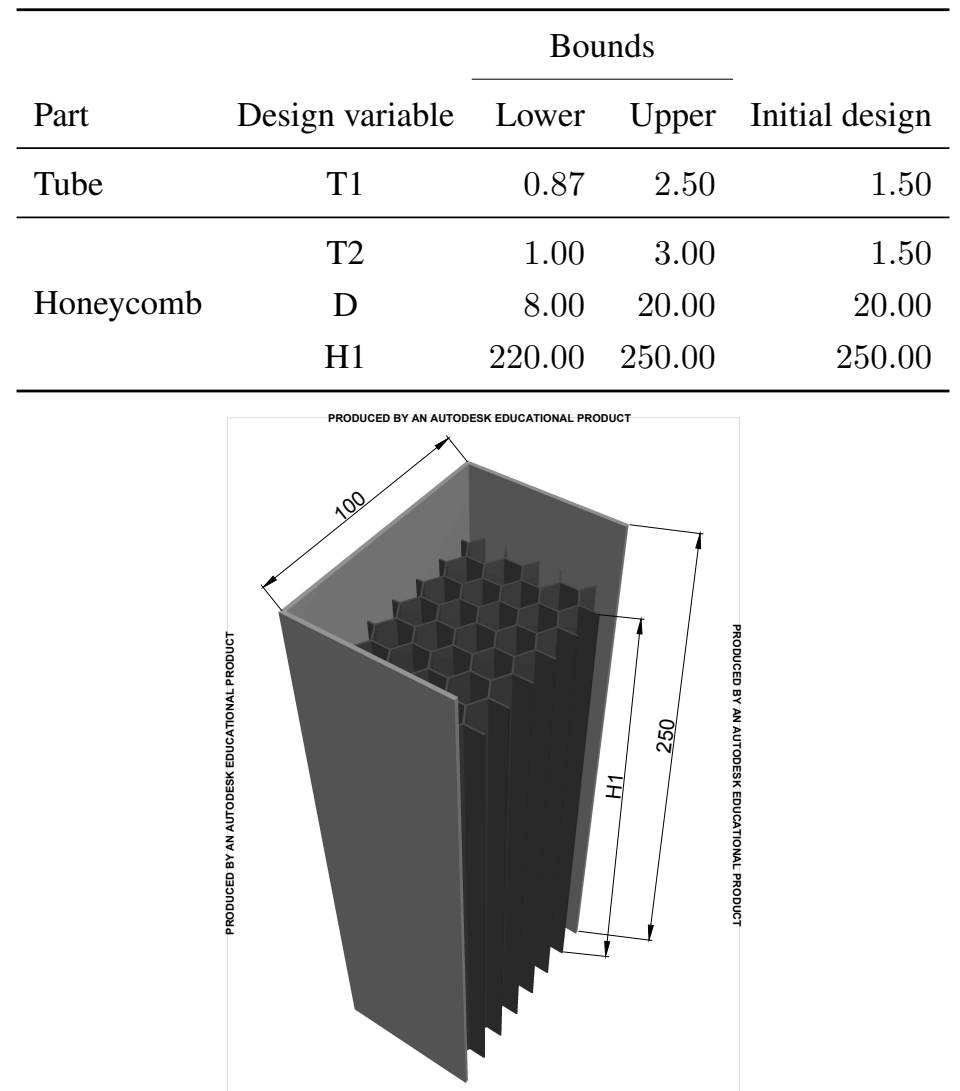

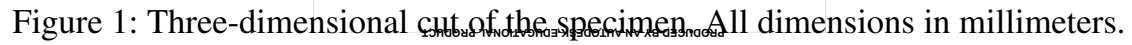

the Johnson-Cook steel model for yield stresses is:

$$
\sigma_{y}=\left[A+B\left(\varepsilon_{p}^{e f f}\right)^{P}\right](1+C \ln \dot{\varepsilon})\left[1-\left(T_{H}\right)^{M}\right]
$$

where $A$ is the yielding stress, $B$ modifies the hardening law amplitude, $C$ affects the strain-rate dependency, $P$ changes the shape of the hardening law, and $M$ adjusts the temperature dependency. Since the temperature dependency of the specimen is not taken into account, $\left(T_{H}\right)^{M}=0$. The parameters for this model were taken from [13], and are offered in table 2.

The inner part of the proposed absorber consists of a honeycomb structure made of a glass-fiber reinforced polyamide (GFRP). The commercial product selected is 


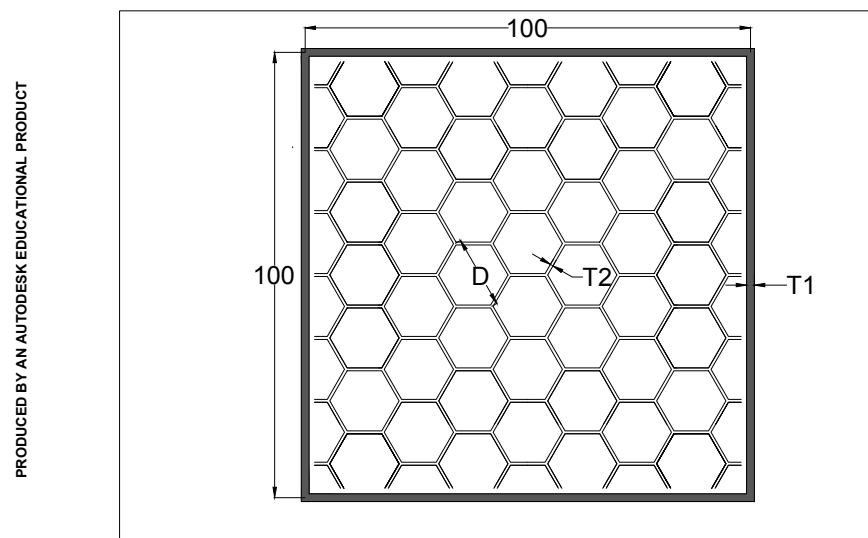

Figure 2: Top view of the specimen. All dimensions in millimeters.

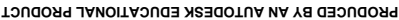

Table 2: S-275 J0H steel properties and Mises plasticity model values for eq. (1).

\begin{tabular}{ccccccccc}
\hline$E$ & $\sigma_{y}^{0}$ & $\nu$ & $\rho$ & A & B & C & P & $\dot{\varepsilon_{0}}$ \\
\hline $210 \mathrm{GPa}$ & $275 \mathrm{MPa}$ & 0.3 & $78.5 \mathrm{kN} / \mathrm{m}^{3}$ & $275 \mathrm{MPa}$ & $50 \mathrm{MPa}$ & 0.03 & 0.4 & 0.0001 \\
\hline
\end{tabular}

named Ultramid A3WG10 BK00564, manufactured by BASF [14]. The properties provided by the manufacturer are offered in table 3 . These properties have also been implemented in the finite element model, as a linear, isotropic, elastic model with fragile failure. The short fibers of the composite are randomly chopped into a polyamide matrix, thus justifying the isotropic behavior. A failure criterion was added to the elastic behavior of the material, removing those elements that overpass a limit strain $\varepsilon_{u}$ of $2.6 \%$.

Table 3: Material properties of Ultramid A3WG10 BK00564.

\begin{tabular}{ccccc}
\hline $\mathrm{E}$ & $\nu$ & $\gamma$ & $\sigma_{u}$ & $\varepsilon_{u}$ \\
\hline $10.16 \mathrm{GPa}$ & 0.4 & $15.15 \mathrm{kN} / \mathrm{m}^{3}$ & $254 \mathrm{MPa}$ & $2.6 \%$ \\
\hline
\end{tabular}

The working principle of this design lies on two different dissipation mechanisms and their interaction. On the one hand, the collapse mechanism of the steel part consists of the formation and development of folds along the metal sheets. Each fold dissipates a limited amount of energy, so it is desirable that the 
steel part develops as many folds as possible. This collapse mechanism has been vastly studied since the 1960 , theoretically, experimentally and computationally. Additional information can be found in references like [15], [16] or [17]. On the other hand, the GFRP honeycomb core shows a fragile fracture behavior and a high stiffness. During the crushing, several sequential fracture planes appear on the reinforcement, and the self-interaction of the dislodged fragments increases the crushing forces of the absorber. Besides, the presence of the core allows more folds to be developed in the steel part, as will be described further on.

As mentioned, the analysis consists of a high speed crushing of the specimen at $17.78 \mathrm{~m} / \mathrm{s}$ up to $18 \mathrm{~cm}$ to prevent the specimen from bottoming-out. If the specimen bottoms-out, the results would be contaminated by the high elastic forces occurring at the end of the crushing process, which do not contribute to the plastic dissipation of energy. The absorber is crushed between two rigid plates, being one of them fixed and the other mobile. Reaction forces are obtained from the fixed plate, and displacements are obtained from the mobile loading plate. Force-displacement curves are obtained from the combination of these two results. According to the specialized literature in crash analysis [18], a standard SAE 600 filter [19] should be applied to the original curves in order to remove high-frequency noise from the signal. The filtered curves will be used to obtain the value of the crashworthiness indicators selected for the parameter study: the specific energy absorption (SEA) and the peak load $\left(P_{\text {peak }}\right)$. Besides, the mass of the specimen is obtained as well.

The absorbed energy can be obtained through a direct integration of the forcedisplacement curve:

$$
E_{a}=\int_{0}^{\delta} F(z) d z,
$$

with $\delta$ being the total axial crushing distance and $F(z)$ the value of the crushing force at the crushing length $z$.

The SEA function is then defined as the ratio of the absorbed energy to the mass of the specimen:

$$
S E A=\frac{E_{a}}{m}
$$

The peak load $P_{\text {peak }}$ is obtained as the maximum value of the crushing force in the curve. A reduction of its value is desirable since crushing forces translate into accelerations on the car occupants. The lower and more constant crushing forces, the less severe injuries for the passengers.

The geometry is introduced in the Abaqus CAE model and carefully meshed. The size of the elements in the outer metal tube must allow the development of folds according to the crushing modes experimentally observed, so the maximum size of these elements is limited. We have adopted a mesh size of $4 \mathrm{~mm}$ for the outer steel part, obtaining satisfactory results. The honeycomb part has been meshed proportionally to the size of the cell, ranging from $1.6 \mathrm{~mm}$ to $3.5 \mathrm{~mm}$, so that every cell wall has at least two elements and no more than six. Both parts are meshed with four-node shell elements with 3 integration points along their thickness and a reduced Simpson integration scheme. 
The simulations are run using Abaqus Explicit [20], which offers quality results even though large plastic deformations, contact and failure occur in the model. Simulations are run on 4 parallel processors, each of which computes a physical part of the model. This configuration has been previously proved as optimum in [21]. Each simulation takes about 1 or 2 hours to complete, these times being strongly dependent on the thicknesses of the parts. No mass scaling has been applied to any part of the model given the high inertial phenomena taking place on it.

\section{Results and discussion}

We have firstly checked that the response of the combined specimen is better than those of both parts analyzed separately and summed afterwards. This fact can be observed in figure 3. The interaction effect accounts for an increase of $12 \%$ in the energy absorbed in this example, with no significant variations in the peak value.

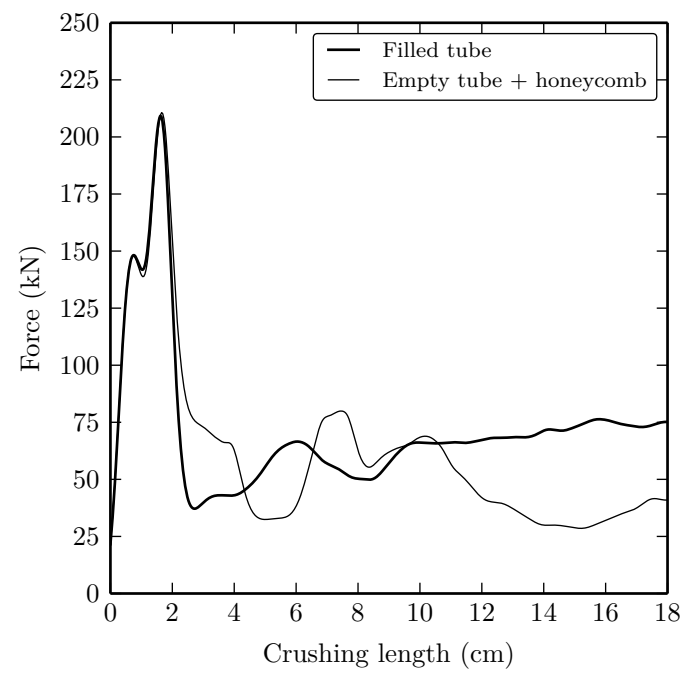

Figure 3: Force-displacement curves of the complete specimen and both parts added together.

This interaction effect constrains the steel part and allows the development of more folds during the crushing of the part, increasing the efficiency of the metal part and developing more stable collapse mechanisms (notice the flattened response of the reinforced tube in figure 3). This is due to the reduction of the buckling lengths in the outer metal sheets due to the elastic contact constrains. These additional folds can be easily observed in figure 4 . 


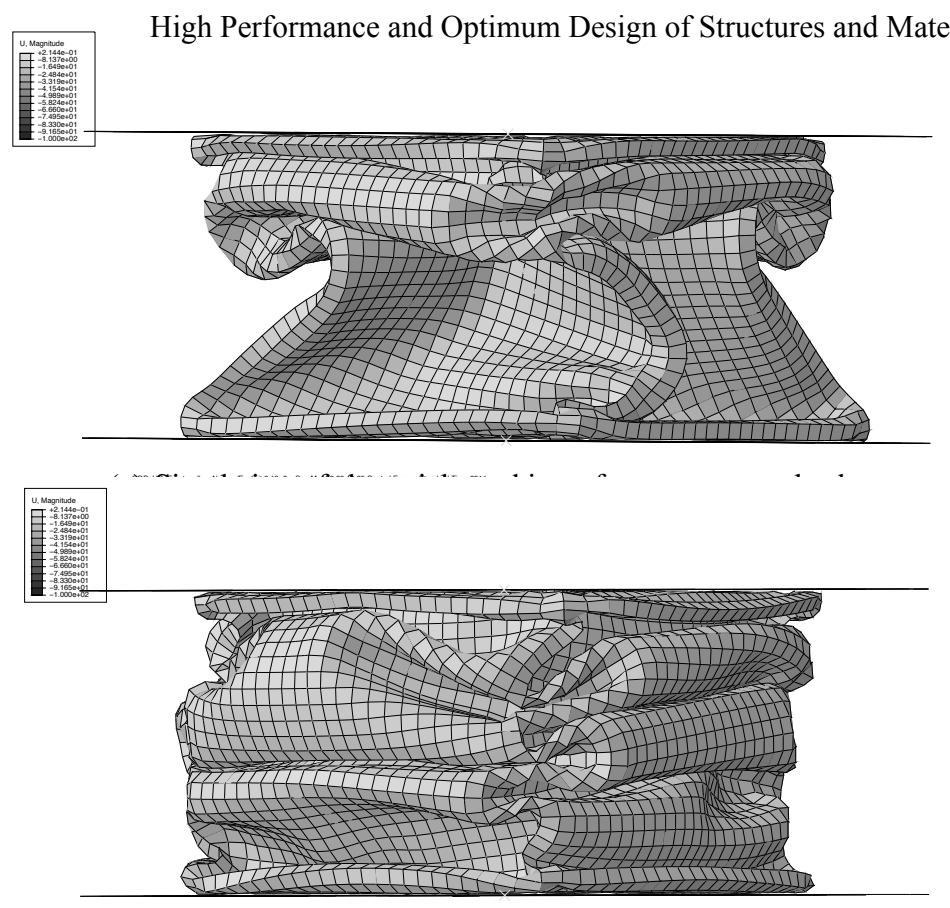

z (b) Simulation ${ }^{\text {so }}$ of the axial crushing of a steel tube reinforced Lawithadinimper GFRP honeycomb at $17.78 \mathrm{~m} / \mathrm{s}$.

Figure 4: Results of the crushing analysis for empty and reinforced tubes.

Once this property is checked, a parameter study has been carried out for each of the 4 variables, and the results of SEA and peak load are offered in figure 5.

At the sight of these results, some information can be extracted. Regarding the cell size $D$, smaller cells increase the peak load since the overall area is increased as well. For cell sizes over $14 \mathrm{~mm}$ this peak load is stabilized. The specific energy absorption has its maximum values for cell sizes in the range from 12 to $16 \mathrm{~mm}$. An optimum design space is found therefore between 14 and $16 \mathrm{~mm}$, with high values of SEA and reduced values of the peak load (figure 5a). The influence of the steel thickness can be observed in figure $5 \mathrm{~b}$. As expected, the peak load increases linearly as the thickness value increases. Regarding the SEA, two ranges must be highlighted: for thicknesses ranging from 1 to $1.50 \mathrm{~mm}$, SEA decreases as the thickness grows. However, from $1.50 \mathrm{~mm}$ on, the specific energy absorption increases almost linearly with the steel thickness. A similar result is observed for the influence of the honeycomb thickness on the crashworthiness indicators (figure 5c). When it comes to the height of the honeycomb core $\mathrm{H} 1$, we can see that it has almost no influence on the specific energy absorption for values within the studied range (figure 5d). However, the initial peak load is greatly affected by this variables. This is due to the overlapping of the steel and GFRP peaks in 


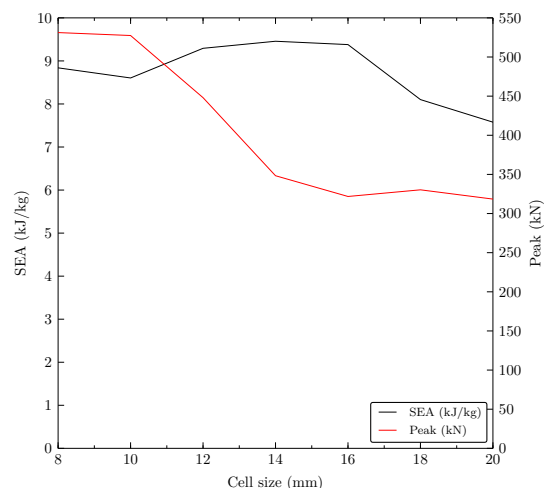

(a) Influence of the cell size (D) on the SEA and the peak load.

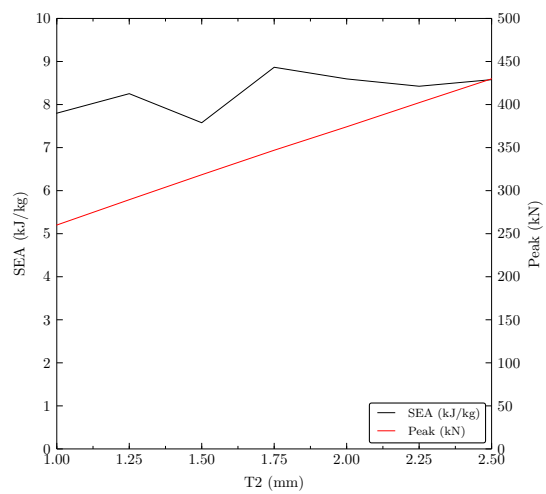

(c) Influence of the GFRP thickness (T2) on the SEA and the peak load.

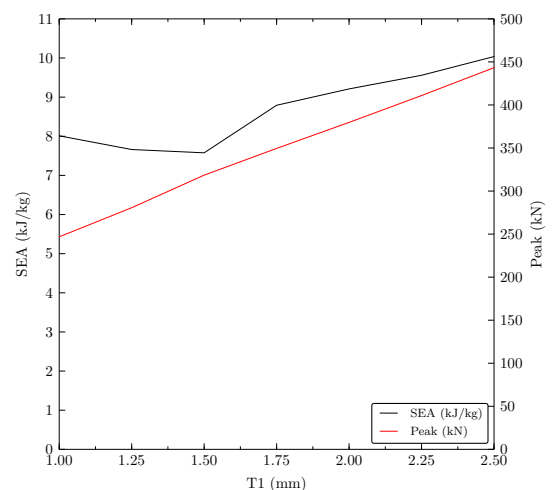

(b) Influence of the steel thickness (T1) on the SEA and the peak load.

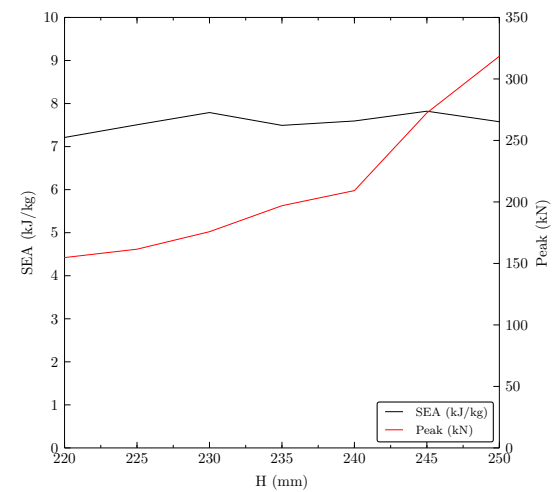

(d) Influence of the honeycomb cell size (D) on the SEA and the peak load.

Figure 5: Results of the parameter study for variables “D”, “T1", “T2” and "H1".

the force-displacement curves obtained in the simulations. This effect appears for honeycomb heights over 240 millimeters.

\section{Conclusions}

A parameter study of an impact absorber made of a steel tube with an inner honeycomb-shaped reinforcement has been carried out. This core is not made of aluminum, as usual, but it is made of a glass-fiber reinforced polyamide. The influence of several geometric parameters on standard crashworthiness indicators has been assessed. 
The glass-fiber reinforced polyamide has shown a good behavior for crashworthiness purposes, and can be considered as a suitable complement to metal absorbers employed nowadays.

The interaction effect between the steel tube and the reinforcement has been quantified as an increment in efficiency by a $12 \%$ in terms of absorbed energy. The inner core constrains the outer tube allowing a more stable collapse mechanism to be developed.

A remarkable influence of the honeycomb cell size has been observed. For values between 14 and 16 millimeters, high values of specific energy absorption are achieved with reduced peaks.

A strong influence of the selected variables on the crashworthiness indicators must be highlighted. As a further work, an optimization could be carried out in order to enhance the capabilities of this absorber.

\section{Acknowledgements}

The research leading to these results has received funding from the Galician Regional Government (Xunta de Galicia) under the plan INCITE09, project reference 09DPI01118PR and GRC2013-056. The authors fully acknowledge the support received.

\section{References}

[1] McFarland, R.K., Hexagonal cell structures under post-buckling axial load. AIAA journal, 1, pp. 1308-1385, 1963.

[2] Hou, S., Li, Q., Long, S., Yang, X. \& Li, W., Design optimization of regular hexagonal thin-walled columns with crashworthiness criteria. Finite Elements in Analysis and Design, 43(6-7), pp. 555-565, 2007.

[3] Aktay, L., Johnson, A. \& Kröplin, B.H., Numerical modelling of honeycomb core crush behaviour. Engineering Fracture Mechanics, 75(9), pp. 26162630, 2008.

[4] Yin, H., Wen, G. \& Gan, N., Crashworthiness design for honeycomb structures under axial dynamic loading. International Journal of Computational Methods, 8(4), pp. 863-877, 2011.

[5] Sun, G., Li, G., Stone, M. \& Li, Q., A two-stage multi-fidelity optimization procedure for honeycomb-type cellular materials. Computational Materials Science, 49(3), pp. 500-511, 2010.

[6] Yin, H., Wen, G., Hou, S. \& Chen, K., Crushing analysis and multiobjective crashworthiness optimization of honeycomb-filled single and bitubular polygonal tubes. Materials and Design, 32(8-9), pp. 4449-4460, 2011.

[7] Schultz, J., Griese, D., Ju, J., Shankar, P., Summers, J. \& Thompson, L., Design of honeycomb mesostructures for crushing energy absorption. Journal of Mechanical Design, Transactions of the ASME, 134(7), 2012. 
[8] Alavi Nia, A. \& Sadeghi, M., The effects of foam filling on compressive response of hexagonal cell aluminum honeycombs under axial loadingexperimental study. Materials and Design, 31(3), pp. 1216-1230, 2010.

[9] Santosa, S. \& Wierzbicki, T., Crash behavior of box columns filled with aluminum honeycomb or foam. Computers and Structures, 68(4), pp. 343$367,1998$.

[10] Zarei, H. \& Kröger, M., Crashworthiness optimization of empty and filled aluminum crash boxes. International Journal of Crashworthiness, 12(3), pp. 255-264, 2007.

[11] European New Car Assessment Programme (Euro NCAP), Frontal impact testing protocol, version 6.0.2, 2013.

[12] Cook, G.R. \& Johson, W.H., A constitutive model and data for metals subjected to large strains, high strain rates and high temperatures. Proceedings of Seventh International Symposium on Ballistics, 1983.

[13] Costas, M., Díaz, J., Romera, L., Hernández, S. \& Tielas, A., Static and dynamic axial crushing analysis of car frontal impact hybrid absorbers. International Journal of Impact Engineering, 62, pp. 166-181, 2013.

[14] BASF, Ultramid (r) A3WG10 BK00564 Polyamide 66. Product information sheet, 2012.

[15] Alexander, J.M., An approximate analysis of the collapse of thin cylindrical shells under axial loading. Quarterly Journal of Mechanics and Applied Mathematics, 13(1), pp. 10-15, 1960.

[16] Abramowicz, W. \& Jones, N., Dynamic progressive buckling of circular and square tubes. International Journal of Impact Engineering, 4(4), pp. 243270, 1986.

[17] Abramowicz, W. \& Jones, N., Transition from initial global bending to progressive buckling of tubes loaded statically and dynamically. International Journal of Impact Engineering, 19(5-6), pp. 415-437, 1997.

[18] Huang, M., Vehicle Crash Mechanics. CRC Press, 2002.

[19] SAE Standard J211: Instrumentation for Impact Test. Technical report, Safety Test Instrumentation Stds Comm, 1995.

[20] Dassault Systèmes, ABAQUS 6.13 Documentation, 2013.

[21] Costas, M., Díaz, J., Romera, L. \& Hernández, A multi-objective surrogatebased optimization of the crashworthiness of a hybrid impact absorber. International Journal of Mechanical Sciences Under review, 2014. 